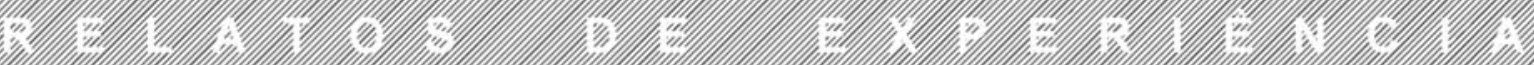

\section{Estratégias de rastreamento e intervenções breves para problemas relacionados ao abuso de álcool entre bombeiros}

\author{
Telmo Mota Ronzani \\ Universidade Federal de Juiz de Fora \\ Thiago Pavin Rodrigues \\ Universidade Federal de São Paulo \\ Andréia Geraldo Batista \\ Corpo de Bombeiros de Minas Gerais \\ Lélio Moura Lourenço \\ Universidade Federal de Juiz de Fora \\ Maria Lucia Oliveira de Souza Formigoni \\ Universidade Federal de São Paulo
}

\begin{abstract}
Resumo
Efeitos deletérios do abuso do álcool são amplamente conhecidos e descritos. A prevenção ao abuso de álcool é particularmente importante em categorias profissionais específicas, por exemplo, trabalhadores submetidos a situações de risco e estresse constantes, como bombeiros. A prevenção pode ser crucial para reduzir possíveis conseqüências do abuso de álcool nessas categorias profissionais. Neste trabalho, ilustramos o uso de uma abordagem em particular, o programa de Triagem e Intervenção Breve (TIB) para bombeiros de Juiz de Fora, MG. A TIB foi realizada em associação com outras avaliações, com um fluxo de intervenção aos bombeiros. Nossa proposta se mostrou factível e sistematizou o atendimento aos mesmos, de forma que permitiu uma integração deste tipo de procedimento com as atividades do setor de saúde. Sugerimos que a TIB, em se tratando de problemas relacionados ao abuso de álcool, se mostrou eficaz neste contexto.
\end{abstract}

Palavras-chave: abuso de álcool; bombeiros; intervenção breve

\begin{abstract}
Screening and brief intervention strategies for problems related to alcohol abuse among firemen. Deleterious effects of alcohol abuse are widely known and described. Prevention of alcohol abuse is particularly important in specific professional categories, i.e. workers subjected to constant risk and stressful situations, like firemen. Prevention may be crucial to reduce possible consequences arising from alcohol abuse in such professional categories. In the present paper we illustrate the use of a particular approach based on prevention, the Screening and Brief Intervention (TIB), on firemen from Juiz de Fora, MG. The TIB has been applied in association with other evaluation strategies and has proved to be viable and efficient as health care prevention in such context.
\end{abstract}

Keywords: alcohol abuse; firemen; brief intervention

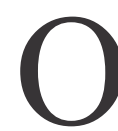
consumo anual médio e o padrão de uso de álcool no Brasil apresentam índices preocupantes, em termos de saúde pública: em média são consumidos 6 litros de álcool puro per capita. Além disso, o padrão de uso de álcool caracteriza-se pelo consumo de altas doses numa mesma ocasião (binge drinking) (Babor et al., 2003).
Associado a isso, observa-se o início de uso de álcool cada vez mais precoce entre jovens (Galduróz, Noto, Fonseca, \& Carlini, 2004), fato que pode gerar diversos problemas de saúde e danos biopsicossociais associados ao consumo em longo prazo (Room \& Babor, 2005).

Estima-se que no Brasil 17,1\% dos homens e 5,7\% das 
mulheres sejam dependentes de álcool, mas que apenas $4 \%$ deste universo tenham freqüentado algum serviço para tratamento (Carlini, Galduroz, Noto, \& Nappo, 2001; Galduróz \& Caetano, 2004). Além do problema da alta prevalência, sabe-se que a dependência ao álcool está em quinto lugar entre os principais problemas de saúde entre a população de 15 a 44 anos em todo o mundo em termos de Anos de Vida Ajustados por Incapacidade (AVAI) (World Health Organization, 1999). Considerando somente alguns países da América Latina, incluindo o Brasil, os transtornos por uso de álcool ocupam a primeira posição em relação aos AVAI (Babor et al., 2003).

Além da indução de dependência, o uso de álcool está relacionado a diversos problemas de saúde. Entre 20 e 58\% de pacientes internados em hospitais gerais apresentam problemas relacionados ao uso de bebidas alcoólicas (Emmen, Schippers, Bleijenberg, \& Wollersheim, 2004; Room \& Babor, 2005). Além disso, estima-se que cerca de $5 \%$ das mortes de pessoas entre 15 e 29 anos, em todo mundo, estejam relacionadas ao uso de álcool (Foxcroft, Ireland, Listter-Sharp, Lowe, \& Breen, 2003). O álcool também está fortemente associado ao alto índice de acidentes de trânsito entre jovens, quedas, afogamentos, acidentes com armas de fogo, suicídios, homicídios, dentre outros problemas sociais relacionados (Beck, Wright, Newman, \& Liese, 1993).

Nos últimos 20 anos houve um progresso considerável no desenvolvimento de instrumentos de triagem associados às técnicas de intervenção breve (IB), direcionados a pacientes em situação de risco relacionados ao consumo de álcool, em serviços de saúde. Os instrumentos de triagem visam detectar problemas de saúde ou fatores de risco em estágios iniciais, antes que eles produzam graves conseqüências (Babor, Higgins-Biddle, Saunders, \& Monteiro, 2001).

Estudos anteriores sugerem que a efetividade da IB e seu desempenho possam ser superiores aos de outras intervenções que demandam maior tempo (Babor \& Higgins-Biddle 2001; Cunningham, Koski-Jannes, Wild, \& Cordingley 2002; Henrique, De Micheli, Lacerda, Lacerda, \& Formigoni 2004; Project MATCH Research Group 1997; WHO ASSIST Working Group, 2002). Além disso, a IB é apontada como uma técnica eficiente, levando em conta os resultados obtidos e o baixo custo para sua execução (Gomel, Wutzke, Hardcastle, Lapsley, \& Reznik 1998).

Um dos instrumentos de triagem para o uso de álcool mais utilizados atualmente em todo mundo é o Alcohol Use Disorders Identification Test (AUDIT). Este é um instrumento desenvolvido pela Organização Mundial de Saúde (OMS), de uso livre, de simples aplicação, contendo apenas 10 perguntas que avaliam o padrão de uso de álcool da população geral. Este instrumento, além de identificar possíveis casos de dependência, permite identificar níveis anteriores de uso de álcool, como uso de risco ou nocivo, que facilita o trabalho preventivo e se torna útil para dar um retorno objetivo à pessoa avaliada (Babor et al., 2001; Ronzani, Ribeiro, Amaral, \& Formigoni, 2005).

Algumas ações de triagem associada a ações de intervenções breves vêm sendo propostas e avaliadas em todo o mundo. A articulação de técnicas de triagem e intervenção breve (TIB) pode também auxiliar na organização do sistema de referência para pessoas que já desenvolveram um transtorno por uso de álcool. No entanto, pouco se tem feito para implementar a TIB para a redução do uso de risco de álcool nos serviços de saúde (Babor \& Higgins-Biddle, 2000). Algumas experiências práticas de disseminação têm sido realizadas em países desenvolvidos; porém, poucas experiências têm sido realizadas quanto à aplicação da TIB em países em desenvolvimento (Babor \& Higgins-Biddle, 2000; Gonçalves et al., 2005; Ronzani et al., 2005; Seppa, 2003).

É muito importante que estas experiências sejam avaliadas e, para isso, a OMS vem desenvolvendo há alguns anos estudos em diversos países para avaliar a implementação de rotinas de triagem e intervenções breves (TIB) em serviços de saúde quanto ao uso de álcool. A ênfase de tais estudos tem sido na avaliação do impacto do treinamento de profissionais de saúde e da educação continuada na mudança de atitudes dos profissionais e na incorporação da TIB na rotina dos serviços de saúde (Babor \& Higgins-Biddle, 2001).

Apesar de mostrar-se eficaz (resultados satisfatórios) e eficiente (baixo custo), a TIB deve ser avaliada em termos de efetividade (aplicabilidade) para se poder chegar a dados mais conclusivos quanto à adequação desta estratégia na prevenção secundária do uso de álcool, em diferentes países. A efetividade da IB foi comprovada quando aplicada por profissionais especializados, porém, ainda é preciso que se avalie seu desempenho quando administrada pelos próprios profissionais dos serviços (Roche \& Freeman, 2004).

Para que uma disseminação de estratégias de implementação de IB seja eficiente, as características de cada serviço e dos profissionais envolvidos precisam ser conhecidas, para que seja possível detectar quais fatores facilitam e quais dificultam a adequada implementação dos programas, visando atingir um bom nível de efetividade (Aalto, Pekuri, \& Seppa, 2005; Aira, Kauhanen, Larivaara, \& Raution, 2004; Babor \& Higgins Biddle, 2001; Babor, Higgins-Biddle, Dauser, Higgins, \& Burleson, 2005).

As pesquisas de avaliação de processo sobre a implementação de rotinas de IB estão sendo realizadas em diversos contextos (Babor \& Higgins-Biddle, 2000; Babor \& Higgins-biddle, 2001; Babor et al., 2003). Porém, a TIB em ambientes de trabalho ainda foi pouco estudada, apesar da importância da implementação de tais práticas nesse contexto. Sabe-se que o uso de álcool está relacionado a vários problemas de trabalho, como acidentes e absenteísmo (Babor et al., 2003; Beck et al., 1993), tornando, portanto, estes locais estratégicos nas ações de prevenção ao uso nocivo de álcool.

Particularmente entre a classe dos bombeiros, devido a este trabalho ser caracterizado como sendo altamente estressante e em constante tensão (Boxer \& Wild, 1998; Dutton, Smolensky, Leach, Lorimor \& Hsi, 1993; Kalimo, Lehtoven, Daleva \& Kuorinka, 1980), constata-se uma alta freqüência de traumatismos e outros acidentes durante o trabalho, além de alta taxa de absenteísmo e uso pesado de álcool (Szubert \& Sobala, 2000).

O objetivo do presente trabalho é apresentar a implementação 
da TIB entre bombeiros do município de Juiz de Fora, Minas Gerais, Brasil.

\section{Local de implantação da estratégia de TIB}

A implementação de estratégias de TIB ocorreu no $4^{\circ}$ Batalhão de Bombeiro Militar de Minas Gerais (4ํㅡㄹ $\mathrm{BB})$, que é uma das cinco Unidades de Execução Operacional do estado de Minas Gerais. Sua área de abrangência compreende toda a Zona da Mata Mineira e Campo das Vertentes, atendendo a aproximadamente 146 municípios, totalizando 2.200.000 habitantes.O efetivo do $4^{\circ} \mathrm{BBM}$ atualmente é composto de 542 militares. A primeira etapa da implementação está ocorrendo na cidade de Juiz de Fora.

Em todo o processo, 303 bombeiros passaram por alguma fase do processo de avaliação de saúde, totalizando $79 \%$ do efetivo da tropa. O perfil dos participantes se configurou como sendo $91 \%$ de homens, com idade média de 33,6 anos $(D P=$ $5,7)$. Em relação ao grau de instrução, $72 \%$ tinham cursado ensino médio e os demais $28 \%$ cursavam, ou tinham cursado, ensino superior. Quanto ao estado civil, 61\% eram casados e 23\% solteiros. O salário médio era de $\mathrm{R} \$ 1.595,00(D P=646,00)$, com tempo médio de trabalho na instituição de 10 anos e 10 meses ( $D P=5$ anos e 10 meses).

A TIB ocorre sob a responsabilidade direta da Seção de Assistência à Saúde (SAS), composta por três oficiais: um médico, um dentista e um psicólogo e por praças auxiliares de saúde. A SAS é o órgão executor do Sistema de Saúde (SiSau) nas Unidades, que presta atendimento a militares e seus dependentes. Suas atividades dividem-se em três áreas: atenção à saúde, prevenção e promoção da saúde.

\section{Divulgação da proposta no Corpo de Bombeiros}

A implantação das estratégias de TIB no Corpo de Bombeiros fez parte de um trabalho mais amplo, direcionado a vários serviços de APS do município de Juiz de Fora, coordenado pela Organização Mundial de Saúde (OMS) (Ronzani et al ., 2005).

A divulgação do projeto ocorreu a partir de um trabalho de sensibilização da comunidade local para a importância da detecção precoce e práticas preventivas ao uso disfuncional de álcool. Neste sentido, a primeira ação foi dirigida aos gestores, profissionais de saúde e pessoas interessadas da comunidade, na forma de um seminário no qual foram resumidamente discutidos os problemas decorrentes do uso abusivo de álcool, sua prevalência e métodos de TIB. Foram convidados para esse seminário vários representantes da comunidade, gestores do sistema de saúde pública e profissionais. Durante o seminário acima referido, os profissionais interessados em participar do treinamento inscreveram-se em uma lista de candidatos. Especificamente no caso dos bombeiros, compareceram ao evento a coordenadora da SAS e um auxiliar de saúde que se interessaram pela proposta e se candidataram a participar do treinamento.

O treinamento, com carga horária de 16 horas, foi realizado conjuntamente com outros profissionais do município e apresentou as técnicas de TIB e estratégias de implementação nos serviços.

\section{Implantação de estratégias de TIB: o caso bombeiros}

Durante todo o processo de implantação da TIB, observouse uma diferença marcante em comparação aos outros serviços de APS. A primeira característica dessa implantação foi o envolvimento direto dos gestores e coordenadores do serviço em relação aos problemas relacionados ao uso de álcool e suas conseqüências para o trabalho dos bombeiros. A partir dessa preocupação, a primeira ação realizada foi conseguir o apoio das instâncias superiores para que as ações de TIB se tornassem "oficializadas" dentro do serviço.

Nesse sentido, foi elaborado um convênio entre a Universidade Federal de Juiz de Fora, envolvendo os pesquisadores e o Corpo de Bombeiros. Neste convênio foram firmadas claramente as responsabilidades de cada parceiro no processo de implantação da TIB.

Um segundo passo para a implantação foi o planejamento de inclusão da TIB dentro de ações mais amplas de saúde. Para tanto, houve a definição de um programa de avaliação em saúde, que estabeleceu que todo o efetivo dos bombeiros seria submetido no período de um ano.

\section{Organização da avaliação do uso de álcool}

Além do uso de álcool, foram incluídas e formalizadas outras avaliações em saúde abrangendo estresse, saúde geral, anamnese médica e saúde mental através da utilização de instrumentos de avaliação psicológica (realizados por acadêmicos do curso de psicologia da UFJF), consultas em saúde (médico e enfermeiro da equipe), vários exames laboratoriais e entrevista psicológica (psicóloga da equipe). As etapas e responsabilidades de cada membro da equipe foram estabelecidas e padronizadas, fato que garantiu o envolvimento de todos os profissionais e uma possível interlocução entre as informações coletadas. Além disso, o retorno do resultado e as ações de prevenção ao uso de álcool foram planejados da seguinte forma:

Etapa 1 - Os bombeiros foram convocados para uma explanação sobre o projeto, na qual a equipe de pesquisadores explicou os objetivos e as etapas do mesmo, visando gerar uma maior adesão dos participantes;

Etapa 2 - Com aqueles bombeiros que aceitaram participar da pesquisa iniciou-se o processo avaliativo pela aplicação coletiva de seis instrumentos: Escala Fatorial de Neuroticismo; Inventário de Habilidades Sociais Del Prette; Inventário de Sintomas de Stress de Lipp; Escalas Beck (de Depressão, Ansiedade, Desesperança e Ideação Suicida); Questionário de Saúde Geral de Goldberg; e AUDIT (Alcohol Use Disorders Idetification Test);

Etapa 3 - Cada indivíduo foi convidado separadamente para uma entrevista semi-estruturada com a oficial psicóloga do batalhão, a fim de reunir informações que pudessem complementar os dados coletados na etapa anterior;

Etapa 4 - Consistiu de uma avaliação médica através de anamnese clínica, realizada pelos médicos e enfermeiros, na qual foram levantadas informações sobre o histórico de doenças e a condição atual de saúde dos bombeiros do batalhão;

Etapa 5 - Foram realizados exames laboratoriais de verificação 
da atividade hepática com o objetivo de coletar dados biológicos sobre uso de álcool dos participantes.

\section{As várias estratégias de intervenção e sua aplicação}

A partir dos resultados encontrados deu-se início às fases de intervenções. Após as análises dos dados do AUDIT foi organizada uma apresentação com o objetivo de dar um retorno (feedback) aos bombeiros sobre os resultados de suas avaliações, de acordo com o setor de trabalho. No momento da apresentação, todos os bombeiros do setor se reuniram com toda a equipe responsável pela avaliação. O retorno foi organizado de forma clara e objetiva, contendo informações relevantes sobre o uso de álcool, explicando as diferenças entre padrões de uso e suas respectivas consequiências para a vida e para o trabalho, bem como mostrando que os profissionais de saúde estavam prontos a atendê-los se precisassem de ajuda, ou de mais informações. O feedback foi baseado no modelo de intervenção breve para usuários de baixo risco de álcool, proposto por Babor e HigginsBiddle (2001).

Paralelamente, os bombeiros foram classificados, de acordo com níveis de uso de álcool, em zonas de risco 1 (abstinentes ou uso de baixo risco), 2 (uso de risco), 3 (uso nocivo) e 4 (provável dependência) conforme sua pontuação no AUDIT. Em seguida, os resultados da entrevista individual foram usados para corroborar ou refutar os resultados anteriores obtidos na aplicação coletiva do instrumento. Finalmente, com dados mais confiáveis, obtivemos um rastreamento mais preciso dos usuários ou abstêmios do $4^{\circ}$ BBM.

Procedeu-se, então, à separação dos bombeiros em três grupos: o primeiro, que englobou os participantes classificados nas zonas 1 e 2; o segundo, que incluiu aqueles classificados na zona 3; e o terceiro grupo, classificado na zona 4. Para aqueles que se encontravam no primeiro e segundo grupos, a orientação básica sobre uso de risco e possíveis danos, de acordo com a quantidade e intensidade de doses ingeridas foi realizada coletivamente. Para aqueles classificados na zona de risco 3, além da participação das orientações básicas, foram realizadas intervenções breves individuais. Para os bombeiros com possível diagnóstico de dependência (zona 4), somou-se ainda uma avaliação mais detalhada e encaminhamento para serviços conveniados, para tratamento.

Uma representação gráfica resumida das estratégias de intervenção adotadas a partir dos dados do AUDIT e da entrevista semi-estruturada encontra-se na Figura 1.

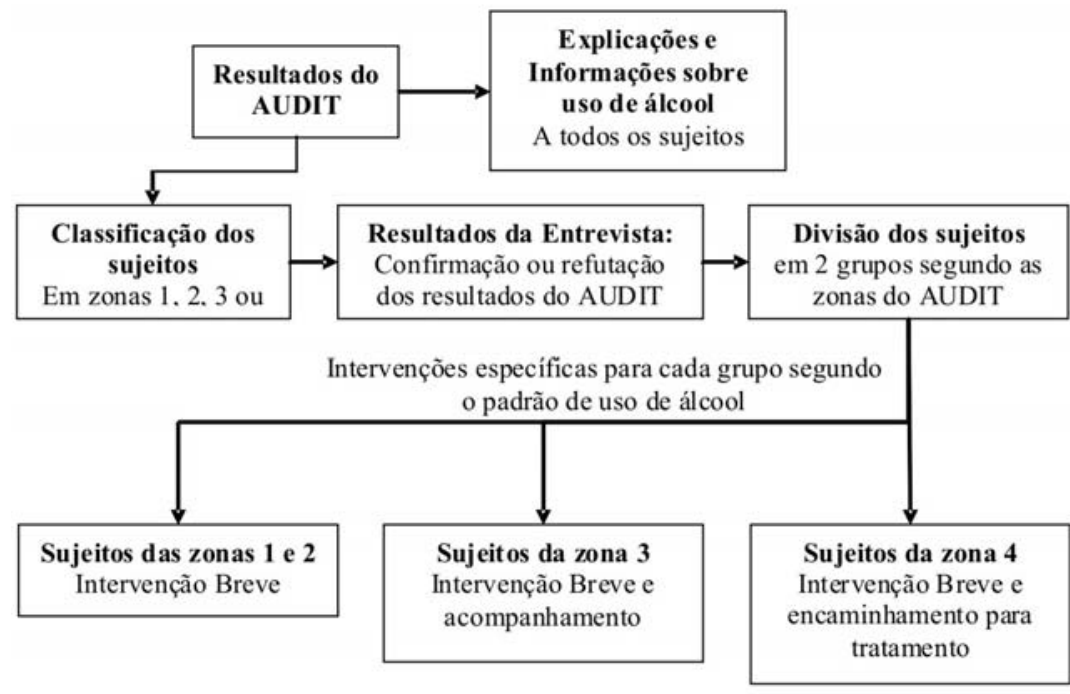

Figura 1. Estratégias para intervenção relativa ao uso de álcool por bombeiros de Juiz de Fora (MG).

\section{Considerações finais}

Ao se considerar o processo de implementação da TIB, de acordo como proposto pelo projeto geral da OMS (Ronzani et al., 2005), pôde-se observar que o projeto no Corpo de Bombeiros, foi promissor e até mesmo se tornou um diferencial, em comparação com os serviços de APS do município (Ronzani, 2005). Este diferencial pode ser justificado principalmente por aspectos organizacionais como: cultura organizacional favorável, comprometimento explícito dos gestores com o projeto (até mesmo do ponto de vista financeiro), organização e sistematização das ações de TIB, definição de responsabilidades e envolvimento dos profissionais. Como conseqüência da qualidade dos resultados alcançados até o momento, existe a proposta de expansão do projeto para outras unidades da região e mesmo do estado.

Os resultados alcançados colaboram para um melhor entendimento sobre os processos de implementação de TIB em serviços de saúde, ressaltando que, além dos aspectos de formação de recursos humanos e mudanças de crenças e atitudes 
sobre o uso de álcool, os aspectos organizacionais devem ser levados em consideração. Um segundo ponto a ser considerado com o presente projeto é que o trabalho de avaliação e prevenção ao abuso de álcool foi implementado como uma ação geral de prevenção à saúde dos bombeiros, dentro de uma perspectiva de qualidade de vida no trabalho. Tal perspectiva facilitou a implementação da TIB, pois evitou a idéia de "combate ao uso de álcool", diminuindo as resistências e aumentando a adesão à proposta. É importante considerar que a hipótese de que os bombeiros teriam algum tipo de resistência ao responder sobre o tema álcool, por se tratar de um ambiente militar e de trabalho, não foi observada ao longo do processo. Isso se deveu principalmente à forma como a proposta foi divulgada e conduzida. Além disso, mesmo considerando que houvesse ainda algum tipo de viés nas respostas, o retorno em grupo e generalizado causaria um impacto nos bombeiros em geral.

Apesar de não configurar o objetivo do presente artigo, que focalizou a avaliação de uma proposta de implementação de um modelo de prevenção ao uso de álcool, ressalta-se a importância de se avaliar o impacto de tal processo como avaliação do custoefetividade como indicadores mais precisos de sucesso da IB nesse contexto.

Portanto, a presente proposta se apresentou como uma importante ação de prevenção ao uso de risco de álcool, sendo uma forma promissora de implementação de ações de TIB, diferenciadas de outros contextos. Mais do que um modelo a ser seguido, a proposta colabora para o melhor entendimento dos vários aspectos a serem considerados e organizados para que ações como estas tenham sucesso.

\section{Referências}

Aira, M., Kauhanen, J., Larivaara, P., \& Rautio, P. (2004). Differences in brief interventions on excessive drinking and smoking by primary care physicians: qualitative study. Preventive Medicine, 38, 473-478.

Babor, T., Caetano, R., Casswell, S., Edwards, G., Giesbrecht, N., Graham, K., Grube, J., Gruenewald, P., Hill, L., Holder, H., Homel, R., Osterberg, E., Rehm, J., Room, R., \& Rossow, I. (2003). Alcohol: no ordinary, no commodity. Research and Public Policy. Nova York: WHO.

Babor, T. F., \& Higgins-Biddle, J. C. (2000). Alcohol screening and brief intervention: dissemination strategies for medical practice and public health. Addiction, 95, 677-686.

Babor, T. F., \& Higgins-Biddle, J. C. (2001). Brief Intervention for Harzardous and Harmful Drinking. A Manual for use in primary care. Genebra: WHO.

Babor, T. F., Higgins-Biddle, J., Dauser, D., Higgins, P., \& Burleson, J. A. (2005). Alcohol screening and brief intervention in primary care settings: implementation models and predictors. Journal of Study on Alcohol, 66, 361-368.

Babor, T. F., Higgins-Biddle, J. C., Saunders, J. B., \& Monteiro, M. G. (2001). AUDIT. The Alcohol Use Disorders Identification Test. Guidelines for use in primary care. Genebra: WHO.

Beck, A. T., Wright, F. D., Newman, C. F., \& Liese, B. S. (1993). Cognitive therapy of substance abuse. Nova York: Guilford.

Boxer, P. A., \& Wild, D. (1993). Psychological distress and alcohol use among fire fighters. Scandinavian Journal of Work, Environment \& Health, 19,
$121-125$.

Carlini, E. A., Galduroz, J. C., Noto, A. R., \& Nappo, S. A. (2001). I Levantamento domiciliar sobre o uso de drogas no Brasil. São Paulo: CEBRID.

Cunningham, J. A., Koski-Jannes, A., Wild, T. C., \& Cordingley, J. (2002). Treating alcohol problems with self-help materials: a population study. Journal of Study on Alcohol, 63, 649-654.

Dutton, L. M., Smolensky, M. H., Leach, C. S., Lorimor, R., \& Hsi, B. P. (1993). Stress levels of ambulance paramedics and fire fighters. Journal of Occupational Medicine, 20, 111-115.

Emmen, M. J., Schippers, G. M., Bleijenberg, G., \& Wollersheim, H. (2004). Effectiveness of opportunistic brief interventions for problem drinking in a general hospital setting: systematic review. British Medical Journal, 328,318 .

Foxcroft, D. R., Ireland, D., Lister-Sharp, D. J., Lowe, G., \& Breen, R. (2003). Longer-term primary prevention for alcohol misuse in young people: a systematic review. Addiction, 98, 397-411.

Galduróz, J. C. F., \& Caetano, R. (2004). Epidemiology of alcohol use in Brazil. Revista Brasileira de Psiquiatria, 26, 3-6.

Galduróz, J. C. F., Noto, A. R., Fonseca, A. M., \& Carlini, E. A. (2004). V Levantamento sobre o uso de drogas psicotrópicas entre estudantes do ensino fundamental e médio da rede pública de ensino nas 27 capitais brasileiras. São Paulo: CEBRID

Gomel, M. K., Wutzke, S. E., Hardcastle, D. M., Lapsley, H., \& Reznik, R. B. (1998). Cost-effectiveness of strategies to market and train primary health care physicians in brief intervention techniques for hazardous alcohol use. Social Science \& Medicine, 47, 203-211.

Gonçalves, P. S., Ronzani, T. M., Bueno, L., Rafael, D., Boerngen-Lacerda, R., Lacerda, L. A. P., \& Formigoni, M. L. O. S. (2005). Primary health care professionals' attitudes, abilities and beliefs regarding early screening and brief intervention for drug abuse. Alcoholism Clinical and Experimental Research, 29, 76A.

Henrique, I. F., De Micheli, D., Lacerda, R. B., Lacerda, L. A., \& Formigoni, M. L. (2004). Validation of version alcohol, smoking, and substance involvment screening test. Revista da Associação Médica Brasileira, 50, 199-206.

Kalimo, R., Lehtonen, A., Daleva, M., \& Kuorinka, I. (1980). Psychological and biochemical strain in firemen's work. Scandinavian Journal of Work, Environment \& Health, 6, 179-187.

Project MATCH Research Group (1997). Matching alcoholism treatments to client heterogeneity: Project MATCH posttreatment drinking outcomes. Journal of Study on Alcohol, 58, 7-29.

Roche, A. M., \& Freeman, T. (2004). Brief interventions: good in theory but weak in practice. Drug and Alcohol Review, 23, 11-18.

Ronzani, T. M., Ribeiro, M. S., Amaral, M. B., \& Formigoni, M. L. O. S. (2005). Implantação de rotinas de rastreamento do uso de risco de álcool e de uma intervenção breve na atenção primária à saúde: dificuldades a serem superadas. Cadernos de Saúde Pública, 21, 852-861.

Room, R., \& Babor, T. (2005). Alcohol and public health. Lancet, 365, 519530 .

Seppa, K. (2003). Development of country-wide strategies for implementing Early Intervention and Brief Alcohol Intervention (EIBI) in primary health care. Norwegian Study on Alcohol \& Drug, 20, 91-96.

Szubert, Z., \& Sobala, W. (2000). Accidents and their health effects in firemen of rescue and firefighting teams. Preventive Medicine, 51, 97-105.

WHO ASSIST Working Group. (2002). The Alcohol, Smoking and Substance Involvement Screening Test (ASSIST): development, reliability and feasibility. Addiction, 97, 1183-1194.

World Health Organization (1999). Global status report on alcohol. Genebra: Autor. 
Telmo Mota Ronzani, doutor em Ciências da Saúde pela Universidade Federal de São Paulo, é professor adjunto no Departamento de Psicologia da Universidade Federal de Juiz de Fora. Endereço para correspondência: Rua Constantino Paleta, 30/404 (Centro); Juiz de Fora, MG; CEP: 36015-450. Tel.: (32) 3218-7437; 88222933. E-mail: telmo.ronzani@ufjf.edu.brou tronzani@hotmail.com

Thiago Pavin Rodrigues, é psicólogo e mestrando em Psicobiologia pela Universidade Federal de São Paulo. E-mail: thiagopavin@yahoo.com.br

Andréia Geraldo Batista, mestranda em Psicologia Social pela Universidade Federal de Minas Gerais, é oficial psicóloga e chefe da Subseção de Psicologia, Assessoria de Assistência à Saúde, Corpo de Bombeiros de Minas Gerais. E-mail: angebatista@gmail.com

Lélio Moura Lourenço, doutor em Psicologia Social pela Pontifícia Universidade Católica de São Paulo, é professor adjunto no Departamento de Psicologia da Universidade Federal de Juiz de Fora. E-mail lelioml@, hotmail.com ou lelio.lourenco@ufjf.edu.br

Maria Lucia Oliveira de Souza Formigoni, doutora em Farmacologia pela Universidade Federal de São Paulo, é professora associada e Chefe do Departamento de Psicobiologia na mesma instituição. E-mail: mlformig@psicobio.epm.brou mlformig@uol.com.br 